\title{
Another class of simple graded Lie conformal algebras that cannot be embedded into general Lie conformal algebras ${ }^{1}$
}

\author{
Yucai Su, Xiaoqing Yue \\ School of Mathematical Sciences, Tongji University, Shanghai 200092, China \\ E-mails: ycsu@tongji.edu.cn, xiaoqingyue@tongji.edu.cn
}

\begin{abstract}
In a previous paper by the authors, we obtain the first example of a finitely freely generated simple $\mathbb{Z}$-graded Lie conformal algebra of linear growth that cannot be embedded into any general Lie conformal algebra. In this paper, we obtain, as a byproduct, another class of such Lie conformal algebras by classifying $\mathbb{Z}$-graded simple Lie conformal algebras $\mathcal{G}=\oplus_{i=-1}^{\infty} \mathcal{G}_{i}$ satisfying the following,

(1) $\mathcal{G}_{0} \cong$ Vir, the Virasoro conformal algebra;

(2) Each $\mathcal{G}_{i}$ for $i \geq-1$ is a Vir-module of rank one.
\end{abstract}

These algebras include some Lie conformal algebras of Block type.

Key words: Lie conformal algebras, the Virasoro conformal algebra, graded Lie conformal algebras, Lie conformal algebras of Block type

Mathematics Subject Classification (2000): 17B10, 17B65, 17B68.

\section{§1. Introduction}

Conformal algebras, first introduced in [15], appear naturally in the context of formal distribution Lie algebras and play important roles in quantum field theory and conformal field theory (e.g., $[4,7,17])$. They also turn out to be effective tools in the study of infinitedimensional Lie or associative algebras satisfying the locality property, and their representations [16].

In recent years, the structure theory, representation theory and cohomology theory of Lie conformal algebras have been extensively studied (e.g., [3, 5-14, 19, 22]). In particular, simple finite Lie conformal algebras were classified in [8], which turn out to be isomorphic either to the Virasoro conformal algebra or the current Lie conformal algebra Cur $\mathfrak{g}$ associated to a simple finite-dimensional Lie algebra $\mathfrak{g}$. Finite irreducible conformal modules over the Virasoro conformal algebra were determined in [6] and of their extensions in [7]. The cohomology theory of conformal algebras with coefficients in an arbitrary module has been developed in $[3,9]$. However, the theory of simple infinite Lie conformal algebras is far from being well developed, it is more complicated than the theory of Lie or associative algebras (e.g., $[13,14])$.

In order to better understand the theory of simple infinite Lie conformal algebras, it is very natural to first study some important examples. It is well-known that the general Lie conformal algebra $g c_{N}$ (which is a simple infinite Lie conformal algebra) plays the same important role in the theory of Lie conformal algebras as the general Lie algebra $g l_{N}$ does in

\footnotetext{
${ }^{1}$ Supported by NSF grant no. 11971350 of China.
} 
the theory of Lie algebras. Thus the study of Lie conformal algebras related to the general Lie conformal algebra $g c_{N}$ has drawn lots of attention in literature (e.g., $\left.[1,2,12,18-21]\right)$. In particular, in [20], we study filtered Lie conformal algebras whose associated graded algebras are isomorphic to that of the general Lie conformal algebra $g c_{1}$, and as a byproduct we obtain the first example of a finitely freely generated simple $\mathbb{Z}$-graded Lie conformal algebra of linear growth that cannot be embedded into a general Lie conformal algebra $g c_{N}$ for any $N$, namely, the Lie conformal algebra gr $g c_{1}$ (the associated graded conformal algebra of $g c_{1}$, which is also called a Lie conformal algebra of Block type), see [20, Theorem 1.1]. Motivated by the facts that a simple Lie conformal algebra of rank one is isomorphic to the Virasoro conformal algebra Vir and that a finite simple Vir-module is of rank one [6-8], in this paper, we study $\mathbb{Z}$-graded Lie conformal algebras $\mathcal{G}=\bigoplus_{i=-1}^{\infty} \mathcal{G}_{i}$ satisfying the following reasonable conditions

(C1) $\mathcal{G}_{0} \cong$ Vir, the Virasoro conformal algebra;

(C2) Each $\mathcal{G}_{i}$ for $i \geq-1$ is a Vir-module of rank one;

(C3) $\mathcal{G}$ is simple.

To state the main result, we first give the following definitions.

Definition 1.1 Let $\alpha \in \mathbb{C}, s=1,2$. Denote by $B(s, \alpha)$ the Lie conformal algebra with $\mathbb{C}[\partial]$-basis $\left\{G_{i} \mid i \in \mathbb{Z}_{\geq-1}\right\}$ and the following $\lambda$-brackets,

$$
\begin{array}{cl}
B(1, \alpha): \quad\left[G_{-1 \lambda} G_{-1}\right]=0, \quad\left[G_{-1 \lambda} G_{0}\right]=(\alpha-\partial) G_{-1}, \quad\left[G_{-1 \lambda} G_{j}\right]=(j+1) G_{j-1}, \quad j \geq 1, \\
{\left[G_{i \lambda} G_{j}\right]=((j-i) \alpha+(i+j+2) \lambda+(i+1) \partial) G_{i+j}, \quad i, j \in \mathbb{Z}_{+},} \\
B(2, \alpha): \quad\left[G_{i \lambda} G_{j}\right]=((j-i) \alpha+(i+j+2) \lambda+(i+1) \partial) G_{i+j}, \quad i, j \in \mathbb{Z}_{\geq-1} .
\end{array}
$$

Definition 1.2 Let $\alpha \in \mathbb{C}$. Denote by $\mathcal{B}(\alpha)=\oplus_{i=-1}^{\infty} \mathcal{B}_{i}$ the $\mathbb{Z}$-graded simple Lie conformal algebra with the $\lambda$-brackets $\left[\mathfrak{b}_{0 \lambda} \mathfrak{b}_{i}\right]=(i \alpha+(i+2) \lambda+\partial) \mathfrak{b}_{i}$ for $i>0$, which satisfies

(i) $\mathcal{B}_{0} \cong$ Vir, the Virasoro conformal algebra,

(ii) $\mathcal{B}_{-1}$ is a Vir-module of rank one,

(iii) Each $\mathcal{B}_{i}$ for $i>0$ is a Vir-module of finite rank,

where $\mathfrak{b}_{0}$ is the $\mathbb{C}[\partial]$-generator of $\mathcal{B}_{0}$ and $\mathfrak{b}_{i}$ is any one of $\mathbb{C}[\partial]$-generators of $\mathcal{B}_{i}$ for $i>0$.

The main result of the present paper is the following.

Theorem 1.3 (1) The Lie conformal algebra $B(s, \alpha)$ is simple for any $\alpha \in \mathbb{C}$ and $s=1,2$. 
(2) For $\alpha_{1}, \alpha_{2} \in \mathbb{C}, s_{1}, s_{2} \in\{1,2\}, B\left(s_{1}, \alpha_{1}\right) \cong B\left(s_{2}, \alpha_{2}\right)$ if and only if $\left(s_{1}, \alpha_{1}\right)=\left(s_{2}, \alpha_{2}\right)$.

(3) Let $\mathcal{G}=\bigoplus_{i=-1}^{\infty} \mathcal{G}_{i}$ be a simple Lie conformal algebra satisfying conditions (C1) and (C2). Then $\mathcal{G} \cong B(s, \alpha)$ for some $\alpha \in \mathbb{C}$ and $s=1,2$.

(4) For any $\alpha \in \mathbb{C}$, the Lie conformal algebra $\mathcal{B}(\alpha)$ does not have a nontrivial representation on any finite $\mathbb{C}[\partial]$-module. In particular, $\mathcal{B}(\alpha)$ is a finitely freely generated simple Lie conformal algebra of linear growth that cannot be embedded into $g c_{N}$ for any $N$.

Therefore, Theorem $1.3(4)$ provides another class $\mathcal{B}(\alpha)$ of finitely freely generated simple $\mathbb{Z}$-graded Lie conformal algebras of linear growth that cannot be embedded into a general Lie conformal algebra $g c_{N}$ for any $N$.

The paper is organized as follows. In section 2, we briefly recall some definitions and preliminary results. In section 3, we first study the structure of the Lie conformal algebra $B(s, \alpha)$, then we give the proof of Theorem $1.3(4)$. In order to classify $\mathbb{Z}$-graded simple Lie conformal algebras $\mathcal{G}$, some technical lemmas were given in section 4 . Then in section 5 , we use these technical lemmas to determine all simple Lie conformal algebras satisfying conditions (C1), (C2), and complete the proof of Theorem 1.3.

Throughout the paper, we denote by $\mathbb{C}, \mathbb{C}^{*}, \mathbb{Z}, \mathbb{Z}_{+}, \mathbb{Z}_{\geq-1}$ the sets of complex numbers, nonzero complex numbers, integers, nonnegative integers and integers greater than -2 respectively.

\section{§2. Definitions and preliminary results}

In this section, we summarize some basic definitions and results concerning Lie conformal algebras. More details can be found in $[3,6,15]$.

Definition 2.1 A Lie conformal algebra is a $\mathbb{C}[\partial]$-module $A$ with a $\lambda$-bracket $\left[\cdot{ }_{\lambda} \cdot\right]$ which defines a $\mathbb{C}$-bilinear map $A \times A \rightarrow A[\lambda]$, where $A[\lambda]=\mathbb{C}[\lambda] \otimes A$ is the space of polynomials of $\lambda$ with coefficients in $A$, such that for $x, y, z \in A$,

$$
\begin{aligned}
& {\left[\partial x_{\lambda} y\right]=-\lambda\left[x_{\lambda} y\right], \quad\left[x_{\lambda} \partial y\right]=(\partial+\lambda)\left[x_{\lambda} y\right] \quad \text { (conformal sesquilinearity), }} \\
& {\left[x_{\lambda} y\right]=-\left[y_{-\lambda-\partial} x\right] \quad(\text { skew-symmetry), }} \\
& {\left[x_{\lambda}\left[y_{\mu} z\right]\right]=\left[\left[x_{\lambda} y\right]_{\lambda+\mu} z\right]+\left[y_{\mu}\left[x_{\lambda} z\right]\right] \quad \text { (Jacobi identity). }}
\end{aligned}
$$

A subset $S \subset A$ is called a generating set if $S$ generates $A$ as a $\mathbb{C}[\partial]$-module. If there exists a finite generating set, then $A$ is called finite. Otherwise, it is called infinite.

For a given Lie conformal algebra $A$, from [15], we know that there is an important Lie algebra associated to it. For each $j \in \mathbb{Z}_{+}$, regarding $\left[a_{\lambda} b\right] \in \mathbb{C}[\lambda] \otimes A$ as a formal polynomial 
in $\lambda$, we can define the $j$ th product $a_{(j)} b$ by the coefficient of $\lambda^{j}$ in $\left[a_{\lambda} b\right]$, i.e. $a_{(j)} b$ for all $a, b \in A$ as follows:

$$
\left[a_{\lambda} b\right]=\sum_{j \in \mathbb{Z}_{+}}\left(a_{(j)} b\right) \frac{\lambda^{j}}{j !} .
$$

Now we can give the definition of this Lie algebra.

Definition 2.2 An annihilation algebra of a Lie conformal algebra $A$ is a Lie algebra with $\mathbb{C}$-basis $\left\{a_{(n)} \mid a \in A, n \in \mathbb{Z}_{+}\right\}$and relations

$$
\left[a_{(m)}, b_{(n)}\right]=\sum_{j \in \mathbb{Z}_{+}}\left(\begin{array}{c}
m \\
j
\end{array}\right)\left(a_{(j)} b\right)_{(m+n-j)}, \quad \partial\left(a_{(n)}\right)=-n a_{(n-1)} .
$$

The Virasoro conformal algebra Vir is the simplest nontrivial Lie conformal algebra. It is a free $\mathbb{C}[\partial]$-module of rank one with generator $L$ and can be defined by

$$
\operatorname{Vir}=\mathbb{C}[\partial] L: \quad\left[L_{\lambda} L\right]=(\partial+2 \lambda) L
$$

It is known that any simple Lie conformal algebra of free rank one over $\mathbb{C}[\partial]$ is isomorphic to Vir [8].

The general Lie conformal algebra $g c_{N}$ can be defined as the infinite rank $\mathbb{C}[\partial]$-module $\mathbb{C}[\partial, x] \otimes g l_{N}$, with the $\lambda$-bracket

$$
\left[f(\partial, x) A_{\lambda} g(\partial, x) B\right]=f(-\lambda, x+\partial+\lambda) g(\partial+\lambda, x) A B-f(-\lambda, x) g(\partial+\lambda, x-\lambda) B A,
$$

for $f(\partial, x), g(\partial, x) \in \mathbb{C}[\partial, x], A, B \in g l_{N}$, where $g l_{N}$ is the space of $N \times N$ matrices, and we have identified $f(\partial, x) \otimes A$ with $f(\partial, x) A$. If we set $J_{A}^{n}=x^{n} A$, then

$$
\left[J_{A \lambda}^{m} J_{B}^{n}\right]=\sum_{s=0}^{m}\left(\begin{array}{c}
m \\
s
\end{array}\right)(\lambda+\partial)^{s} J_{A B}^{m+n-s}-\sum_{s=0}^{n}\left(\begin{array}{c}
n \\
s
\end{array}\right)(-\lambda)^{s} J_{B A}^{m+n-s},
$$

for $m, n \in \mathbb{Z}_{+}, A, B \in g l_{N}$, where $\left(\begin{array}{c}m \\ s\end{array}\right)=m(m-1) \cdots(m-s+1) / s$ ! if $s \geq 0$ and $\left(\begin{array}{l}m \\ s\end{array}\right)=0$ otherwise, is the binomial coefficient.

Definition 2.3 A module over a Lie conformal algebra $A$ is a $\mathbb{C}[\partial]$-module $M$ with a $\lambda$ action $\cdot_{\lambda} \cdot A \times M \rightarrow M[[\lambda]]$, where $M[[\lambda]]$ is the set of formal power series of $\lambda$ with coefficients in $M$, such that for $x, y \in A, v \in M$,

$$
\begin{aligned}
& x_{\lambda}\left(y_{\mu} v\right)-y_{\mu}\left(x_{\lambda} v\right)=\left[x_{\lambda} y\right]_{\lambda+\mu} v, \\
& (\partial x)_{\lambda} v=-\lambda x_{\lambda} v, \quad x_{\lambda}(\partial v)=(\partial+\lambda) x_{\lambda} v .
\end{aligned}
$$

An $A$-module $M$ is called conformal if $x_{\lambda} v \in M[\lambda]$ for $x \in A, v \in M$ and finite if $M$ is finitely generated over $\mathbb{C}[\partial]$. 
According to [6], we know that all free nontrivial conformal Vir-modules of rank one over $\mathbb{C}[\partial]$ are $M_{\Delta, \alpha}$ for $\Delta, \alpha \in \mathbb{C}$, where

$$
M_{\Delta, \alpha}=\mathbb{C}[\partial] v: \quad L_{\lambda} v=(\alpha+\partial+\Delta \lambda) v
$$

The module $M_{\Delta, \alpha}$ is irreducible if and only if $\Delta \neq 0$.

\section{§3. Graded Lie conformal algebras $B(s, \alpha)$}

It is straightforward to verify that (1.1) indeed defines Lie conformal algebras $B(1, \alpha)$ and $B(2, \alpha)$. Furthermore, the annihilation algebra of $B(2, \alpha)$ is the Lie algebra $\mathcal{A}=$ $\operatorname{span}_{\mathbb{C}}\left\{G_{i, m} \mid i, m \in \mathbb{Z}_{\geq-1}\right\}$ with Lie brackets

$$
\left[G_{i, m}, G_{j, n}\right]=(j-i) \alpha G_{i+j, m+n+1}+((j+1)(m+1)-(n+1)(i+1)) G_{i+j, m+n} .
$$

When $\alpha=0$, this Lie algebra has close relation to the Block-type Lie algebras studied in $[12,19]$.

Now we study the structure of the Lie conformal algebra $B(s, \alpha)$ for $\alpha \in \mathbb{C}$ and $s=1,2$. First we need some definitions. For any $x \in B(s, \alpha)$, we define the operator $(\operatorname{ad} x)_{\lambda}$ : $B(s, \alpha) \rightarrow B(s, \alpha)[\lambda]$ such that $(\operatorname{ad} x)_{\lambda}(y)=\left[x_{\lambda} y\right]$ for any $y \in B(s, \alpha)$. An element $x \in$ $B(s, \alpha)$ is locally nilpotent if for any $y \in B(s, \alpha)$, there exists $1 \leq n \in \mathbb{Z}_{+}$such that $(\operatorname{ad} x)_{\lambda}^{n}(y)=0$. We have

Lemma 3.1 The set of locally nilpotent elements of $B(s, \alpha)$ is equal to $\mathbb{C}[\partial] G_{-1}$.

Proof. Denote by $\mathcal{N}$ the set of locally nilpotent elements of $B(s, \alpha)$. First by (1.1) and conformal sesquilinearity, we have the following for $a_{0}(\partial), c_{j}(\partial) \in \mathbb{C}[\partial]$,

$$
\left[a_{0}(\partial) G_{-1 \lambda} c_{j}(\partial) G_{j}\right]= \begin{cases}(\alpha-\partial) a_{0}(-\lambda) c_{j}(\partial+\lambda) G_{-1} & \text { if } s=1, j=0 \\ (j+1) a_{0}(-\lambda) c_{j}(\partial+\lambda) G_{j-1} & \text { if } s=1,1 \leq j \in \mathbb{Z} \\ (j+1)(\alpha+\lambda) a_{0}(-\lambda) c_{j}(\partial+\lambda) G_{j-1} & \text { if } s=2,0 \leq j \in \mathbb{Z}\end{cases}
$$

From this and using conformal sesquilinearity, we immediately obtain that $\mathbb{C}[\partial] G_{-1} \subset \mathcal{N}$.

Now let $x=\sum_{i=-1}^{\infty} b_{i}(\partial) G_{i} \in \mathcal{N}$, suppose $\max \left\{i \mid b_{i}(\partial) \neq 0\right\}=i_{0}$. If $i_{0} \geq 0$, by (1.1), we have $\left[G_{i_{0} \lambda} G_{j}\right]=\left(\left(j-i_{0}\right) \alpha+\left(i_{0}+j+2\right) \lambda+\left(i_{0}+1\right) \partial\right) G_{i_{0}+j}$ for $j \in \mathbb{Z}_{+}$. Then applying $(\operatorname{ad} x)_{\lambda}^{n}$ to $G_{j}$, we can obtain that the coefficient of $G_{n i_{0}+j}$ in the expression of $(\operatorname{ad} x)_{\lambda}^{n}\left(G_{j}\right)$ is nonzero for any $1 \leq n \in \mathbb{Z}_{+}$and $j \in \mathbb{Z}_{+}$. This is a contradiction with $x \in \mathcal{N}$. Therefore we must have $i_{0}=-1$, then the lemma follows.

Proof of Theorem 1.3(1) and (2). (1) Let $J$ be a nonzero ideal of $B(s, \alpha)$ for some $\alpha \in \mathbb{C}$ and $s=1,2$. Then there exists at least one nonzero element $x=\sum_{j=-1}^{m} b_{j}(\partial) G_{j} \in J$ for 
some $b_{j}(\partial) \in \mathbb{C}[\partial]$, where $m \in \mathbb{Z}_{+}$such that $b_{m}(\partial) \neq 0$. We claim that $a_{0}(\partial) G_{-1} \in J$ for some nonzero $a_{0}(\partial) \in \mathbb{C}[\partial]$. If $m=-1$, we immediately have the claim. Otherwise, we can apply the operator $\left(\operatorname{ad} G_{-1}\right)_{\lambda}^{m+1}$ to $x$, we have the following for $b_{m}(\partial) \in \mathbb{C}[\partial]$,

$$
J \ni\left(\operatorname{ad} G_{-1}\right)_{\lambda}^{m+1}(x)= \begin{cases}(m+1) !(\alpha-\partial) b_{m}(\partial+\lambda) G_{-1} & \text { if } s=1 \\ (m+1) !(\alpha+\lambda)^{m} b_{m}(\partial+\lambda) G_{-1} & \text { if } s=2\end{cases}
$$

Then we inductively deduce from (3.1) that all $G_{j} \in J$ for $j \in \mathbb{Z}_{\geq-1}$, i.e., $J=B(s, \alpha)$. Therefore, $B(s, \alpha)$ is simple.

(2) It is obvious that the sufficient condition holds. We only need to prove the necessary condition. For $\alpha_{1}, \alpha_{2} \in \mathbb{C}, s_{1}, s_{2} \in\{1,2\}$, we suppose $B\left(s_{1}, \alpha_{1}\right) \cong B\left(s_{2}, \alpha_{2}\right),\left\{G_{i} \mid i \in \mathbb{Z}_{\geq-1}\right\}$ and $\left\{G_{i}^{\prime} \mid i \in \mathbb{Z}_{\geq-1}\right\}$ are the $\mathbb{C}[\partial]$-bases of $B\left(s_{1}, \alpha_{1}\right)$ and $B\left(s_{2}, \alpha_{2}\right)$ respectively. By (1.1), we can immediately conclude that $s_{1}=s_{2}$.

First suppose $s_{1}=1$. Let $\varphi: B\left(1, \alpha_{1}\right) \longrightarrow B\left(1, \alpha_{2}\right)$ be an isomorphism. By Lemma 3.1, we can assume $\varphi\left(G_{-1}\right)=a(\partial) G_{-1}^{\prime}$ and $\varphi\left(G_{0}\right)=\sum_{i=-1}^{\infty} b_{i}(\partial) G_{i}^{\prime}$ for some $a(\partial), b_{i}(\partial) \in \mathbb{C}[\partial]$ with $i \in \mathbb{Z}_{\geq-1}$. Applying the isomorphism $\varphi$ to the both sides of $\left[G_{-1_{\lambda}} G_{0}\right]=\left(\alpha_{1}-\partial\right) G_{-1}$, comparing the coefficients of $G_{i-1}^{\prime}$ for $1 \leq i \in \mathbb{Z}$ and $G_{-1}^{\prime}$ respectively, we can deduce that $b_{i}(\partial)=0$ for $1 \leq i \in \mathbb{Z}$ and

$$
\left(\alpha_{2}-\partial\right) a(-\lambda) b_{0}(\lambda+\partial)=\left(\alpha_{1}-\partial\right) a(\partial)
$$

By (1.1), we have $\left[G_{0 \lambda} G_{0}\right]=(2 \lambda+\partial) G_{0}$. Applying the isomorphism $\varphi$ to this equation, then comparing the coefficients of $G_{0}^{\prime}$, we can obtain that

$$
b_{0}(-\lambda) b_{0}(\lambda+\partial)=b_{0}(\partial)
$$

Comparing the degrees of $\lambda$ in (3.4) and using the fact that $\varphi$ is an isomorphism, we have $b_{0}(\partial)=1$. Then by (3.3), we can conclude that $\alpha_{1}=\alpha_{2}$.

Now suppose $s_{1}=2$. Similarly, if $B\left(2, \alpha_{1}\right) \cong B\left(2, \alpha_{2}\right)$, we can also obtain that $\alpha_{1}=\alpha_{2}$. Therefore, if $\varphi: B\left(s, \alpha_{1}\right) \longrightarrow B\left(s, \alpha_{2}\right)$ is an isomorphism, then $\left(s_{1}, \alpha_{1}\right)=\left(s_{2}, \alpha_{2}\right)$.

In order to prove Theorem $1.3(4)$, we need some preparations. Assume $V$ is a finitely freely $\mathbb{C}[\partial]$-generated nontrivial $\mathcal{B}(\alpha)$-module. Regarding $V$ as a module over Vir, by $[6$, Theorem 3.2(1)], we can choose a composition series,

$$
V=V_{N} \supset V_{N-1} \supset \cdots \supset V_{1} \supset V_{0}=0
$$

such that for each $i=1,2, \ldots, N$, the composition factor $\bar{V}_{i}=V_{i} / V_{i-1}$ is either a rank one free module $M_{\Delta_{i}, \beta_{i}}$ with $\Delta_{i} \neq 0$, or else a 1-dimensional trivial module $\mathbb{C}_{\beta_{i}}$ with trivial $\lambda$-action and with $\partial$ acting as the scalar $\beta_{i}$. Denote by $\bar{v}_{i}$ a $\mathbb{C}[\partial]$-generator of $\bar{V}_{i}$ and $v_{i} \in V_{i}$ the 
preimage of $\bar{v}_{i}$. Then $\left\{v_{i} \mid 1 \leq i \leq N\right\}$ is a $\mathbb{C}[\partial]$-generating set of $V$, such that the $\lambda$-action of $\mathfrak{b}_{0}$ on $v_{i}$ is a $\mathbb{C}[\lambda, \partial]$-combination of $v_{1}, \ldots, v_{i}$.

Lemma 3.2 For all $i \gg 0$, the $\lambda$-action of $\mathfrak{b}_{i}$ on $v_{1}$ is trivial, namely, $\mathfrak{b}_{i_{\lambda}} v_{1}=0$.

Proof. Assume $i \gg 0$ is fixed and suppose $\mathfrak{b}_{i \lambda} v_{1} \neq 0$, and let $k_{i} \geq 1$ be the largest integer such that $\mathfrak{b}_{i \lambda} v_{1} \not \subset V_{k_{i}-1}[\lambda]$. We consider the following possibilities.

Case 1: $V_{1}=M_{\Delta_{1}, \beta_{1}}, \bar{V}_{k_{i}}=M_{\Delta_{k_{i}}, \beta_{k_{i}}}$.

We can write

$$
\mathfrak{b}_{i \lambda} v_{1} \equiv p_{i}(\lambda, \partial) v_{k_{i}}\left(\bmod V_{k_{i}-1}[\lambda]\right) \text { for some } p_{i}(\lambda, \partial) \in \mathbb{C}[\lambda, \partial]
$$

Applying the operator $\mathfrak{b}_{0 \mu}$ to (3.5), we obtain

$$
\left(\beta_{k_{i}}+\partial+\Delta_{k_{i}} \mu\right) p_{i}(\lambda, \mu+\partial)=(i \alpha+(1+i) \mu-\lambda) p_{i}(\lambda+\mu, \partial)+\left(\beta_{1}+\lambda+\partial+\Delta_{1} \mu\right) p_{i}(\lambda, \partial)
$$

Letting $\partial=0$, we have

$$
p_{i}(\lambda, \mu)=\frac{1}{\beta_{k_{i}}+\Delta_{k_{i}} \mu}\left((i \alpha+(1+i) \mu-\lambda) p_{i}(\lambda+\mu, 0)+\left(\beta_{1}+\lambda+\Delta_{1} \mu\right) p_{i}(\lambda, 0)\right) .
$$

Using this in (3.6) with $\lambda=i \alpha+(1+i) \mu$ and $\partial=-\beta_{k_{i}}-\Delta_{k_{i}} \mu$, we can deduce that

$$
\begin{aligned}
& (i+1)\left(\left(\Delta_{k_{i}}+1\right) \mu+\beta_{k_{i}}\right) p_{i}\left(\left(i+1-\Delta_{k_{i}}\right) \mu-\beta_{k_{i}}+i \alpha, 0\right) \\
& \quad=\left(\left(i+1-\Delta_{1} \Delta_{k_{i}}\right) \mu+\beta_{1}-\beta_{k_{i}} \Delta_{1}\right) p_{i}((i+1) \mu+i \alpha, 0) .
\end{aligned}
$$

Suppose $p_{i}(\lambda, 0)$ has degree $m_{i}$. Comparing the coefficients of $\mu^{m_{i}+1}$ in the above equation, we obtain (note that the following equation does not depend on the coefficients of $p_{i}(\lambda, \mu)$ )

$$
(i+1)\left(\Delta_{k_{i}}+1\right)\left(i+1-\Delta_{k_{i}}\right)^{m_{i}}=\left(i+1-\Delta_{1} \Delta_{k_{i}}\right)(i+1)^{m_{i}} .
$$

When $i$ is sufficiently large, one can easily see that (3.8) cannot hold if $m_{i}>1$ (note that $\Delta_{1}, \Delta_{k_{i}} \neq 0$, and there are only finitely many choices for what $\Delta_{k_{i}}$ can be, since $\left.1 \leq k_{i} \leq N\right)$. Thus $m_{i} \leq 1$ if $i \gg 0$. Then from (3.7), we obtain that $p_{i}(\lambda, \mu)$ is a polynomial of degree $\leq 1$. Thus suppose $p_{i}(\lambda, \mu)=a_{i, 0}+a_{i, 1} \lambda+a_{i, 2} \mu$. Then by comparing the coefficients of $\lambda \mu$, $\mu \partial$ and $\mu$ respectively in (3.6), we immediately have $p_{i}(\lambda, \mu)=0$.

Case 2: $V_{1}=\mathbb{C}_{\beta_{1}}, \bar{V}_{k_{i}}=M_{\Delta_{k_{i}}, \beta_{k_{i}}}$.

In this case, we can still assume (3.5). Applying the operator $\mathfrak{b}_{0 \mu}$ to (3.5), we obtain

$$
p_{i}(\lambda, \mu+\partial)\left(\beta_{k_{i}}+\partial+\Delta_{k_{i}} \mu\right)=(i \alpha+(1+i) \mu-\lambda) p_{i}(\lambda+\mu, \partial) .
$$

Taking $\mu=\partial=0$, we get $p_{i}(\lambda, 0)=0$. Then letting $\partial=0$, we obtain $p_{i}(\lambda, \mu)=0$.

Case 3: $V_{1}=M_{\Delta_{1}, \beta_{1}}, \bar{V}_{k_{i}}=\mathbb{C}_{\beta_{k_{i}}}$.

In this case, since $\partial$ acts on $\bar{v}_{k_{i}}$ as the scalar $\beta_{k_{i}}$, i.e., $\partial v_{k_{i}} \equiv \beta_{k_{i}} v_{k_{i}}\left(\bmod V_{k_{i}-1}[\lambda]\right)$, we can write

$$
\mathfrak{b}_{i \lambda} v_{1} \equiv p_{i}(\lambda) v_{k_{i}}\left(\bmod V_{k_{i}-1}[\lambda]\right) \text { for some } p_{i}(\lambda) \in \mathbb{C}[\lambda]
$$


Applying the operator $\mathfrak{b}_{0 \mu}$ to (3.10), we obtain

$$
0=(i \alpha+(1+i) \mu-\lambda) p_{i}(\lambda+\mu)+\left(\beta_{1}+\lambda+\partial+\Delta_{1} \mu\right) p_{i}(\lambda) .
$$

By comparing the coefficients of $\partial$, we immediately get $p_{i}(\lambda)=0$.

Case 4: $V_{1}=\mathbb{C}_{\beta_{1}}, \bar{V}_{k_{i}}=\mathbb{C}_{\beta_{k_{i}}}$.

As above, we can still assume (3.10). Applying the operator $\mathfrak{b}_{0 \mu}$ to (3.10), in this case we obtain

$$
0=(i \alpha+(1+i) \mu-\lambda) p_{i}(\lambda+\mu) .
$$

It is obvious that $p_{i}(\lambda)=0$.

Finally we can give the proof of Theorem 1.3 (4). By induction on $j \leq N$, we obtain $\mathfrak{b}_{i \lambda} v_{j}=0$, i.e., the $\lambda$-action of $\mathfrak{b}_{i}$ is trivial. From this, we immediately obtain that the $\lambda$ action of $\mathcal{B}(\alpha)$ on $V$ is trivial since $\mathcal{B}(\alpha)$ is a simple Lie conformal algebra. Then Theorem 1.3 (4) follows.

\section{§4. Some technical lemmas}

Let $\mathcal{G}=\bigoplus_{i=-1}^{\infty} \mathcal{G}_{i}$ be a simple Lie conformal algebra satisfying conditions (C1) and (C2). The main problem to be addressed in this paper is to classify these $\mathbb{Z}$-graded Lie conformal algebras. In order to solve the main problem, we need some preparations. Since $\mathcal{G}=\oplus_{i=-1}^{\infty} \mathcal{G}_{i}$ satisfying conditions $(\mathrm{C} 1)$ and $(\mathrm{C} 2)$, denote by $\mathfrak{g}_{i}$ a $\mathbb{C}[\partial]$-generator of $\mathcal{G}_{i}$, then $\left\{\mathfrak{g}_{i} \mid i \in \mathbb{Z}_{\geq-1}\right\}$ is a $\mathbb{C}[\partial]$-generating set of $\mathcal{G}$. By $(2.9)$, we can suppose

$$
\begin{aligned}
& {\left[\mathfrak{g}_{-1 \lambda} \mathfrak{g}_{-1}\right]=0} \\
& {\left[\mathfrak{g}_{0 \lambda} \mathfrak{g}_{j}\right]=\left(\alpha_{j}+\partial+\Delta_{j} \lambda\right) \mathfrak{g}_{j},} \\
& {\left[\mathfrak{g}_{i \lambda} \mathfrak{g}_{j}\right]=g_{i, j}(\lambda, \partial) \mathfrak{g}_{i+j}}
\end{aligned}
$$

where $\alpha_{j}, \Delta_{j} \in \mathbb{C}$ for $j \in \mathbb{Z}_{\geq-1}$ and $g_{i, j}(\lambda, \partial) \in \mathbb{C}[\lambda, \partial]$ for $i, j \in \mathbb{Z}_{\geq-1}$ are polynomials of $\lambda$ and $\partial$. From (4.3), we can see that $\mathfrak{g}_{i+j}$ can be generated by $\mathfrak{g}_{i}$ and $\mathfrak{g}_{j}$ for $i, j \in \mathbb{Z}_{\geq-1}$. It is very natural to firstly consider $g_{i, j}(\lambda, \partial)$ for $i=0, j \in \mathbb{Z}_{\geq-1}$ and $i=1, j=-1$. This is also the aim of this section. Based on this, in the next section we will determine all $g_{i, j}(\lambda, \partial)$ for $i, j \in \mathbb{Z}_{\geq-1}$ in the proof of Theorem $1.3(3)$. Since $\mathcal{G}$ is simple, we also know that

$$
\left[\mathfrak{g}_{-1 \lambda} \mathfrak{g}_{j}\right] \neq 0 \quad \text { for } \quad 1 \leq j \in \mathbb{Z}
$$

Lemma 4.1 In (4.2), we have $\Delta_{0}=2$ and $\alpha_{j}=j \alpha_{1}$ for $j \in \mathbb{Z}_{\geq-1}$; in particular, we get $\alpha_{0}=0$. Thus for $j \in \mathbb{Z}_{\geq-1}$ we can suppose

$$
\begin{aligned}
& g_{0,0}(\lambda, \partial)=\partial+2 \lambda, \\
& g_{0, j}(\lambda, \partial)=j \alpha_{1}+\partial+\Delta_{j} \lambda .
\end{aligned}
$$


Proof. From $\mathcal{G}_{0} \cong$ Vir, by (2.5), we can conclude that $\Delta_{0}=2, \alpha_{0}=0$ in (4.2), thus (4.5) holds. Now applying the operator $\mathfrak{g}_{0_{\mu}}$ to (4.3), using the Jacobi identity $\left[\mathfrak{g}_{0 \mu}\left[\mathfrak{g}_{i \lambda} \mathfrak{g}_{j}\right]\right]=$ $\left[\left[\mathfrak{g}_{0 \mu} \mathfrak{g}_{i}\right]_{\lambda+\mu} \mathfrak{g}_{j}\right]+\left[\mathfrak{g}_{i \lambda}\left[\mathfrak{g}_{0 \mu} \mathfrak{g}_{j}\right]\right]$ and comparing the coefficients of $\mathfrak{g}_{i+j}$ for $i, j \in \mathbb{Z}_{\geq-1}$, we obtain

$$
\begin{gathered}
\left(\alpha_{j+i}+\partial+\Delta_{j+i} \mu\right) g_{i, j}(\lambda, \mu+\partial)-\left(\alpha_{j}+\partial+\lambda+\Delta_{j} \mu\right) g_{i, j}(\lambda, \partial) \\
=\left(\alpha_{i}-\lambda+\left(\Delta_{i}-1\right) \mu\right) g_{i, j}(\lambda+\mu, \partial)
\end{gathered}
$$

Now taking $\mu=0$ in (4.7), one can immediately get that $\left(\alpha_{j+i}-\alpha_{j}-\alpha_{i}\right) g_{i, j}(\lambda, \partial)=0$ for all $i, j \in \mathbb{Z}_{\geq-1}$. Then the lemma follows.

In order to determine all the polynomials $g_{i, j}(\lambda, \partial)$, we would first like to deal with the case with $i=1$ and $j=-1$. There is no need to compute $g_{-1,1}(\lambda, \partial)$ as it can be determined from $g_{1,-1}(\lambda, \partial)$ by skew-symmetry. Comparing the coefficients of $\mathfrak{g}_{j+1}$ on both sides of the Jacobi identity $\left[\mathfrak{g}_{0 \lambda}\left[\mathfrak{g}_{1 \mu} \mathfrak{g}_{j}\right]\right]=\left[\left[\mathfrak{g}_{0 \lambda} \mathfrak{g}_{1}\right]_{\lambda+\mu} \mathfrak{g}_{j}\right]+\left[\mathfrak{g}_{1 \mu}\left[\mathfrak{g}_{0 \lambda} \mathfrak{g}_{j}\right]\right]$, by (4.3) and Lemma 4.1, we have

$$
\begin{gathered}
\left((j+1) \alpha_{1}+\partial+\Delta_{j+1} \lambda\right) g_{1, j}(\mu, \lambda+\partial)-\left(j \alpha_{1}+\partial+\mu+\Delta_{j} \lambda\right) g_{1, j}(\mu, \partial) \\
=\left(\alpha_{1}-\mu+\left(\Delta_{1}-1\right) \lambda\right) g_{1, j}(\lambda+\mu, \partial) .
\end{gathered}
$$

Taking $\partial=0$ and $j=-1$, by Lemma 4.1 , we obtain

$$
\begin{aligned}
g_{1,-1}(\mu, \lambda)= & \frac{1}{2 \lambda}\left(\left(\alpha_{1}-\mu+\left(\Delta_{1}-1\right) \lambda\right) g_{1,-1}(\lambda+\mu, 0)\right. \\
& \left.-\left(\alpha_{1}-\mu-\Delta_{-1} \lambda\right) g_{1,-1}(\mu, 0)\right) .
\end{aligned}
$$

Similarly, applying the operator $\mathfrak{g}_{-1_{\lambda}}$ to $\left[\mathfrak{g}_{1 \mu} \mathfrak{g}_{j}\right]$, using the Jacobi identity and comparing the coefficients of $\mathfrak{g}_{j}$, by (4.3) and Lemma 4.1, we obtain

$$
\begin{gathered}
g_{-1, j+1}(\lambda, \partial) g_{1, j}(\mu, \lambda+\partial)-g_{-1, j}(\lambda, \partial+\mu) g_{1, j-1}(\mu, \partial) \\
=\left(j \alpha_{1}+\partial+\Delta_{j}(\lambda+\mu)\right) g_{-1,1}(\lambda,-\lambda-\mu) .
\end{gathered}
$$

Setting $j=-1$ and replacing $\partial, \lambda$ by $-\lambda,-\mu-\partial$ in (4.10) respectively, by (2.2), (4.1), (4.3) and Lemma 4.1, we can deduce that

$$
\left(\alpha_{1}-\mu-\partial\right) g_{1,-1}(\mu, 0)=\left(\alpha_{1}-\mu+\left(\Delta_{-1}-1\right) \partial\right) g_{1,-1}(\mu, \partial) \text {. }
$$

Using (4.9) in the above formula, we get

$$
\begin{aligned}
& \left(\alpha_{1}-\mu+\left(\Delta_{1}-1\right) \partial\right)\left(\alpha_{1}-\mu+\left(\Delta_{-1}-1\right) \partial\right) g_{1,-1}(\mu+\partial, 0) \\
& \quad=\left(2 \partial\left(\alpha_{1}-\mu-\partial\right)+\left(\alpha_{1}-\mu-\Delta_{-1} \partial\right)\left(\alpha_{1}-\mu+\left(\Delta_{-1}-1\right) \partial\right)\right) g_{1,-1}(\mu, 0)
\end{aligned}
$$


Letting $\mu=0$ implies the following,

$$
\begin{aligned}
\left(\alpha_{1}\right. & \left.+\left(\Delta_{1}-1\right) \partial\right)\left(\alpha_{1}+\left(\Delta_{-1}-1\right) \partial\right) g_{1,-1}(\partial, 0) \\
& =\left(2 \partial\left(\alpha_{1}-\partial\right)+\left(\alpha_{1}-\Delta_{-1} \partial\right)\left(\alpha_{1}+\left(\Delta_{-1}-1\right) \partial\right)\right) g_{1,-1}(0,0)
\end{aligned}
$$

Taking $\partial=-\mu$ in (4.12), and replacing $\mu$ by $\partial$, we obtain

$$
\begin{gathered}
\left(-2 \partial \alpha_{1}+\left(\alpha_{1}-\Delta_{-1} \partial\right)\left(\alpha_{1}+\left(\Delta_{-1}-1\right) \partial\right)\right) g_{1,-1}(\partial, 0) \\
=\left(\alpha_{1}-\Delta_{1} \partial\right)\left(\alpha_{1}-\Delta_{-1} \partial\right) g_{1,-1}(0,0) .
\end{gathered}
$$

Since $g_{1,-1}(\lambda, \partial)$ is a polynomial of $\lambda$ and $\partial$, we can write $g_{1,-1}(\partial, 0)=\sum_{i=0}^{m} a_{1,-1}^{i} \partial^{i}$ for some $a_{1,-1}^{i} \in \mathbb{C}$ for $0 \leq i \leq m$. We need to consider whether or not $\alpha_{1} \neq 0$. First assume $\alpha_{1} \neq 0$. If $a_{1,-1}^{0}=0$, then comparing the degrees of $\partial$ on both sides of (4.14), we immediately have $g_{1,-1}(\partial, 0)=0$, which implies that $g_{1,-1}(\lambda, \partial)=0$ by $(4.9)$, a contradiction with (4.4). If $a_{1,-1}^{0} \neq 0$, we have the following.

Lemma 4.2 Assume $\alpha_{1} \neq 0$ and $a_{1,-1}^{0} \neq 0$. Then $\Delta_{-1}=0, \Delta_{1}=3$ or $\Delta_{-1}=1, \Delta_{1}=3$. Furthermore,

$$
g_{1,-1}(\lambda, \partial)= \begin{cases}a_{1,-1}^{0} & \text { if } \Delta_{-1}=0, \Delta_{1}=3, \\ \frac{a_{1,-1}^{0}}{\alpha_{1}}\left(\alpha_{1}-\lambda-\partial\right) & \text { if } \quad \Delta_{-1}=1, \Delta_{1}=3 .\end{cases}
$$

Proof. Since $\alpha_{1} \neq 0$ and $g_{1,-1}(0,0)=a_{1,-1}^{0} \neq 0$, comparing the degrees of $\partial$ on both sides of (4.14), we get $a_{1,-1}^{i}=0$ for $2 \leq i \leq m$. And we need to consider the following possibilities.

Case 1: $\Delta_{-1}=0$.

If $\Delta_{-1}=0$, by (4.14), we must have $\Delta_{1}=3$ and $g_{1,-1}(\partial, 0)=a_{1,-1}^{0}$. Therefore, by (4.9) we have $g_{1,-1}(\lambda, \partial)=a_{1,-1}^{0}$, i.e., the first case of (4.15) holds.

Case 2: $\Delta_{-1}=1$.

In this case, (4.14) shows that $\Delta_{1}=3$ and $a_{1,-1}^{1}=-\frac{a_{1,-1}^{0}}{\alpha_{1}}$. Using this in (4.9), we get the second case of (4.15).

Case $3: \Delta_{-1} \neq 0$ and $\Delta_{-1} \neq 1$.

If $\Delta_{-1} \neq 0$ and $\Delta_{-1} \neq 1$, comparing the degrees of $\partial$ on both sides of (4.14), we know that $g_{1,-1}(\partial, 0)=g_{1,-1}(0,0) \neq 0$. Then comparing the coefficients of $\partial^{i}$ for $i=1,2$ on both 
sides of (4.14) respectively, we have $\Delta_{-1}+\Delta_{1}=3$ and $\Delta_{-1}+\Delta_{1}=1$, a contradiction. Hence the lemma follows.

Now we deal with the case $\alpha_{1}=0$. If $a_{1,-1}^{0} \neq 0$, taking $\alpha_{1}=0$ in (4.13) and (4.14) and comparing the coefficients of $\partial^{2}$ on both sides respectively, we can deduce that $\Delta_{-1}=0$, $\Delta_{1}=3$ and $g_{1,-1}(\partial, 0)=a_{1,-1}^{0}$. Then by (4.9) we immediately obtain the following.

Lemma 4.3 If $\alpha_{1}=0$ and $a_{1,-1}^{0} \neq 0$, then $\Delta_{-1}=0, \Delta_{1}=3$ and $g_{1,-1}(\lambda, \partial)=a_{1,-1}^{0}$.

Now we can consider the most complicated case that $\alpha_{1}=0$ and $a_{1,-1}^{0}=0$. In this case (4.13) and (4.14) turn into

$$
\begin{aligned}
& \left(\Delta_{1}-1\right)\left(\Delta_{-1}-1\right) g_{1,-1}(\partial, 0)=0 \\
& \Delta_{-1}\left(1-\Delta_{-1}\right) g_{1,-1}(\partial, 0)=0 .
\end{aligned}
$$

Lemma 4.4 If $\alpha_{1}=0$ and $a_{1,-1}^{0}=0$, then we can deduce that $\Delta_{-1}=1, \Delta_{1}=3$ and

$$
g_{1,-1}(\lambda, \partial)=a_{1,-1}^{1}(\lambda+\partial) \quad \text { for } \quad a_{1,-1}^{1} \in \mathbb{C}^{*}
$$

Proof. By (4.4) and (4.17), our discussion will be divided into the following two cases.

Case 1: $\Delta_{-1}=0$.

From (4.16), it follows that $\left(\Delta_{1}-1\right) g_{1,-1}(\partial, 0)=0$. If $\Delta_{1} \neq 1$, then it is obvious that $g_{1,-1}(\partial, 0)=0$. This together with $(4.9)$ shows that $g_{1,-1}(\lambda, \partial)=0$, i.e., a contradiction with (4.4). Now we suppose $\Delta_{1}=1$. Taking $\lambda=-\mu \neq 0$ in (4.9), using the fact that $a_{1,-1}^{0}=0$, we obtain

$$
g_{1,-1}(\mu,-\mu)=-\frac{1}{2} g_{1,-1}(\mu, 0) .
$$

In addition, (4.11) shows that

$$
g_{1,-1}(\mu, \partial)=g_{1,-1}(\mu, 0) \quad \text { for } \partial \neq-\mu \text {. }
$$

Letting $\alpha_{1}=0, \Delta_{-1}=0$ and $\Delta_{1}=1$ in $(4.12)$, we have $\mu g_{1,-1}(\mu+\partial, 0)=(\mu-2 \partial) g_{1,-1}(\mu, 0)$ for $\mu+\partial \neq 0$. Then setting $\mu=1$ and $\partial=-\mu+1$ in this formula respectively, we get

$$
\begin{aligned}
& g_{1,-1}(\mu, 0)=(3-2 \mu) g_{1,-1}(1,0) \quad \text { for } \quad \mu \neq 0 \\
& \mu g_{1,-1}(1,0)=(3 \mu-2) g_{1,-1}(\mu, 0)
\end{aligned}
$$

Inserting (4.21) into (4.22) gives that $(\mu-1)^{2} g_{1,-1}(1,0)=0$ for $\mu \neq 0$. It leads to $g_{1,-1}(1,0)=$ 0 . Then (4.19), (4.20) together with (4.21) show that $g_{1,-1}(\lambda, \partial)=0$, i.e., we also get a 
contradiction with (4.4).

Case 2: $\Delta_{-1}=1$.

Taking $\alpha_{1}=0$ and $\Delta_{-1}=1$ in (4.12), we have

$$
\mu\left(\left(\Delta_{1}-1\right) \partial-\mu\right) g_{1,-1}(\mu+\partial, 0)=(\mu+\partial)(2 \partial-\mu) g_{1,-1}(\mu, 0)
$$

Letting $\partial=-\mu+1$ in the above formula, then replacing $\mu$ by $\partial$, we can obtain

$$
(2-3 \partial) g_{1,-1}(\partial, 0)=\partial\left(\Delta_{1}-1-\Delta_{1} \partial\right) g_{1,-1}(1,0)
$$

Recall that $g_{1,-1}(\partial, 0)=\sum_{i=1}^{m} a_{1,-1}^{i} \partial^{i}$. Comparing the coefficients of $\partial^{i}$ for $1 \leq i \leq m+1$ on both sides of (4.23), we deduce that $a_{1,-1}^{i}=0$ for $2 \leq i \leq m, a_{1,-1}^{1}=g_{1,-1}(1,0)$ and $\left(\Delta_{1}-3\right) a_{1,-1}^{1}=0$. Thus we have that $g_{1,-1}(\partial, 0)=a_{1,-1}^{1} \partial$ and $\left(\Delta_{1}-3\right) a_{1,-1}^{1}=0$. If $a_{1,-1}^{1}=0$, it follows that $g_{1,-1}(\partial, 0)=0$, then (4.9) leads to $g_{1,-1}(\lambda, \partial)=0$. Therefore, by (4.4) and (4.9), we have $g_{1,-1}(\lambda, \partial)=a_{1,-1}^{1}(\lambda+\partial) \neq 0$. And this lemma holds.

\section{§5. Classification of graded Lie conformal algebras}

In this section, we determine all $g_{i, j}(\lambda, \partial)$ for $i, j \in \mathbb{Z}_{\geq-1}$, so that we can classify $\mathbb{Z}$-graded simple Lie conformal algebras $\mathcal{G}=\bigoplus_{i=-1}^{\infty} \mathcal{G}_{i}$.

Proof of Theorem 1.3(3). Applying $\mathfrak{g}_{-1_{\lambda}}$ to $\left[\mathfrak{g}_{0 \mu} \mathfrak{g}_{j}\right]$, by (2.3), we have the Jacobi identity $\left[\mathfrak{g}_{-1_{\lambda}}\left[\mathfrak{g}_{0 \mu} \mathfrak{g}_{j}\right]\right]=\left[\left[\mathfrak{g}_{-1_{\lambda}} \mathfrak{g}_{0}\right]_{\lambda+\mu} \mathfrak{g}_{j}\right]+\left[\mathfrak{g}_{0 \mu}\left[\mathfrak{g}_{-1_{\lambda}} \mathfrak{g}_{j}\right]\right]$. Then comparing the coefficients of $\mathfrak{g}_{j-1}$, by (4.3) and Lemma 4.1, we obtain

$$
\begin{gathered}
\left(j \alpha_{1}+\lambda+\partial+\Delta_{j} \mu\right) g_{-1, j}(\lambda, \partial)-\left((j-1) \alpha_{1}+\partial+\Delta_{j-1} \mu\right) g_{-1, j}(\lambda, \partial+\mu) \\
=\left(\alpha_{1}+\lambda-\left(\Delta_{-1}-1\right) \mu\right) g_{-1, j}(\lambda+\mu, \partial) .
\end{gathered}
$$

Taking $j=1$ in (4.10), we have

$$
\begin{gathered}
g_{-1,2}(\lambda, \partial) g_{1,1}(\mu, \lambda+\partial)-g_{-1,1}(\lambda, \partial+\mu) g_{1,0}(\mu, \partial) \\
=\left(\alpha_{1}+\partial+\Delta_{1}(\lambda+\mu)\right) g_{-1,1}(\lambda,-\lambda-\mu)
\end{gathered}
$$

By skew-symmetry, we have $g_{-1,1}(\lambda, \partial)=-g_{1,-1}(-\lambda-\partial, \partial)$, so for convenience, we suppose $a_{-1,1}^{0}=-a_{1,-1}^{0}$. By Lemma 4.2-4.4, we need to consider the following three cases.

Case 1: $\Delta_{-1}=0, \Delta_{1}=3$ and $g_{1,-1}(\lambda, \partial)=-a_{-1,1}^{0} \neq 0$.

Since we have skew-symmetry, by Lemma 4.1 , we have $g_{-1,1}(\lambda, \partial)=a_{-1,1}^{0}$ and $g_{1,0}(\lambda, \partial)=$ $-\alpha_{1}+3 \lambda+2 \partial$. Then (5.2) turns into

$$
g_{-1,2}(\lambda, \partial) g_{1,1}(\mu, \lambda+\partial)=3 a_{-1,1}^{0}(\lambda+2 \mu+\partial)
$$


Note that both $g_{-1,2}(\lambda, \partial)$ and $g_{1,1}(\lambda, \partial)$ are polynomials of $\lambda$ and $\partial$, so by comparing the coefficients on both sides of the above formula, we can deduce that

$$
\begin{aligned}
& g_{-1,2}(\lambda, \partial)=a_{-1,2}^{0}, \\
& g_{1,1}(\lambda, \partial)=\frac{3 a_{-1,1}^{0}}{a_{-1,2}^{0}}(2 \lambda+\partial),
\end{aligned}
$$

where $a_{-1,2}^{0} \in \mathbb{C}^{*}$. Setting $j=2$ in $(5.1)$ and noting that $g_{-1,2}(\lambda, \partial)=a_{-1,2}^{0} \neq 0$, we get

$$
\Delta_{2}=\Delta_{1}+1=4
$$

By (4.10), (5.1) and (5.3)-(5.5), we can inductively deduce that

$$
\begin{aligned}
& \Delta_{j}=j+2, \\
& g_{-1, j}(\lambda, \partial)=a_{-1, j}^{0}, \\
& g_{1, j}(\lambda, \partial)=\frac{a_{-1,1}^{0}}{2 a_{-1, j+1}^{0}}(j+2)\left((j-1) \alpha_{1}+(j+3) \lambda+2 \partial\right),
\end{aligned}
$$

where $1 \leq j \in \mathbb{Z}$ and $a_{-1, j}^{0} \in \mathbb{C}^{*}$.

Now we want to determine $g_{j, 2}(\lambda, \partial)$ for $2 \leq j \in \mathbb{Z}$. Comparing the coefficients of $\mathfrak{g}_{j+2}$ on both sides of $\left[\mathfrak{g}_{j_{\lambda}}\left[\mathfrak{g}_{1 \mu} \mathfrak{g}_{1}\right]\right]=\left[\left[\mathfrak{g}_{j_{\lambda}} \mathfrak{g}_{1}\right]_{\lambda+\mu} \mathfrak{g}_{1}\right]+\left[\mathfrak{g}_{1_{\mu}}\left[\mathfrak{g}_{j_{\lambda}} \mathfrak{g}_{1}\right]\right]$, we obtain

$$
\begin{aligned}
& g_{j, 2}(\lambda, \partial) g_{1,1}(\mu, \lambda+\partial)-g_{j, 1}(\lambda, \partial+\mu) g_{1, j+1}(\mu, \partial) \\
& =g_{j, 1}(\lambda,-\lambda-\mu) g_{j+1,1}(\lambda+\mu, \partial) .
\end{aligned}
$$

From this, using (5.8) and skew-symmetry, we obtain, for $1 \leq j \in \mathbb{Z}$,

$$
g_{j, 2}(\lambda, \partial)=\frac{-a_{-1,1}^{0} a_{-1,2}^{0}}{6 a_{-1, j+1}^{0} a_{-1, j+2}^{0}}(j+2)(j+3)\left((j-2) \alpha_{1}-(j+4) \lambda-(j+1) \partial\right) .
$$

Finally, we can determine all the polynomials $g_{j, i}(\lambda, \partial)$ as follows. Noting from $\left[\mathfrak{g}_{j_{\lambda}}\left[\mathfrak{g}_{\mu_{\mu}} \mathfrak{g}_{i}\right]\right]=$ $\left[\left[\mathfrak{g}_{j_{\lambda}} \mathfrak{g}_{1}\right]_{\lambda+\mu} \mathfrak{g}_{i}\right]+\left[\mathfrak{g}_{1 \mu}\left[\mathfrak{g}_{j_{\lambda}} \mathfrak{g}_{i}\right]\right]$, we obtain

$$
\begin{gathered}
g_{j, i+1}(\lambda, \partial) g_{1, i}(\mu, \lambda+\partial)-g_{j, i}(\lambda, \partial+\mu) g_{1, j+i}(\mu, \partial) \\
=g_{j, 1}(\lambda,-\lambda-\mu) g_{j+1, i}(\lambda+\mu, \partial) .
\end{gathered}
$$

By (5.8)-(5.10), we can inductively deduce, for $1 \leq i \in \mathbb{Z}, 1 \leq j \in \mathbb{Z}$,

$$
\begin{aligned}
g_{j, i}(\lambda, \partial)= & \frac{-a_{-1,1}^{0} a_{-1,2}^{0} \cdots a_{-1, i}^{0}}{a_{-1, j+1}^{0} a_{-1, j+2}^{0} \cdots a_{-1, j+i}^{0}} \times \frac{(j+2)(j+3) \cdots(j+i+1)}{(i+1) !} \\
& \times\left((j-i) \alpha_{1}-(j+i+2) \lambda-(j+1) \partial\right)
\end{aligned}
$$


In order for the polynomials $g_{j, i}(\lambda, \partial)$ to have some suitable forms, for $1 \leq j \in \mathbb{Z}$, we replace $\mathfrak{g}_{j}$ by $\mathfrak{g}_{j}^{\prime}=\frac{(j+1) !}{a_{-1,1}^{0} a_{-1,2}^{0} \cdots a_{-1, j}^{0}} \mathfrak{g}_{j}$, so that $g_{-1, j}(\lambda, \partial)$ and $g_{j, i}(\lambda, \partial)$ have the following forms,

$$
\begin{aligned}
& g_{-1, j}(\lambda, \partial)=j+1 \text { for } 1 \leq j \in \mathbb{Z}, \\
& g_{j, i}(\lambda, \partial)=(i-j) \alpha_{1}+(i+j+2) \lambda+(j+1) \partial \text { for } 1 \leq i \in \mathbb{Z}, 1 \leq j \in \mathbb{Z} .
\end{aligned}
$$

Since in this case $\Delta_{-1}=0$, using (4.6) and skew-symmetry, we obtain $g_{-1,0}(\lambda, \partial)=\alpha_{1}-\partial$. By (4.6) and (5.6), it is not hard to check that the second equation of the above also holds for $i=0$ or $j=0$. Therefore, in this case we obtain $\mathcal{G} \cong B(1, \alpha)$ for some $\alpha \in \mathbb{C}$.

Case 2: $\Delta_{-1}=1, \Delta_{1}=3, \alpha_{1}=0$ and $g_{1,-1}(\lambda, \partial)=a_{-1,1}^{1}(\lambda+\partial)$, where $a_{-1,1}^{1} \in \mathbb{C}^{*}$.

In this case, using skew-symmetry, we obtain that $g_{-1,1}(\lambda, \partial)=a_{-1,1}^{1} \lambda$ and $g_{1,0}(\lambda, \partial)=$ $3 \lambda+2 \partial$. Then (5.2) turns into

$$
g_{-1,2}(\lambda, \partial) g_{1,1}(\mu, \lambda+\partial)=3 a_{-1,1}^{1} \lambda(\lambda+2 \mu+\partial) .
$$

Since $g_{-1,2}(\lambda, \partial)$ and $g_{1,1}(\lambda, \partial)$ are polynomials of $\lambda$ and $\partial$, we get from the above formula,

$$
\begin{aligned}
& g_{-1,2}(\lambda, \partial)=a_{-1,2}^{1} \lambda, \\
& g_{1,1}(\lambda, \partial)=\frac{3 a_{-1,1}^{1}}{a_{-1,2}^{1}}(2 \lambda+\partial),
\end{aligned}
$$

for some $a_{-1,2}^{1} \in \mathbb{C}^{*}$. Taking $j=2, \Delta_{-1}=1, \Delta_{1}=3$ and $\alpha_{1}=0$ in (5.1), and noting that $a_{-1,2}^{1} \neq 0$, we get

$$
\Delta_{2}=4
$$

Therefore, by (4.10), (5.1) and (5.12)-(5.14), we can inductively prove that

$$
\begin{aligned}
& \Delta_{j}=j+2, \\
& g_{-1, j}(\lambda, \partial)=a_{-1, j}^{1} \lambda, \\
& g_{1, j}(\lambda, \partial)=\frac{a_{-1,1}^{1}}{2 a_{-1, j+1}^{1}}(j+2)((j+3) \lambda+2 \partial),
\end{aligned}
$$

where $1 \leq j \in \mathbb{Z}$ and $a_{-1, j}^{1} \in \mathbb{C}^{*}$. Similar to Case 1 , the above three formulae together with (5.10) inductively show the following,

$$
\begin{aligned}
g_{j, i}(\lambda, \partial)= & \frac{a_{-1,1}^{1} a_{-1,2}^{1} \cdots a_{-1, i}^{1}}{a_{-1, j+1}^{1} a_{-1, j+2}^{1} \cdots a_{-1, j+i}^{1}} \times \frac{(j+2)(j+3) \cdots(j+i+1)}{(i+1) !} \\
& \times((j+i+2) \lambda+(j+1) \partial),
\end{aligned}
$$


for $1 \leq i \in \mathbb{Z}$ and $1 \leq j \in \mathbb{Z}$. Replace $\mathfrak{g}_{j}$ by $\mathfrak{g}_{j}^{\prime}=\frac{(j+1) !}{a_{-1,1}^{1} a_{-1,2}^{1} \cdots a_{-1, j}^{1}} \mathfrak{g}_{j}$ for $1 \leq j \in \mathbb{Z}$, so that $g_{-1, j}(\lambda, \partial)$ and $g_{j, i}(\lambda, \partial)$ have the following forms,

$$
\begin{aligned}
& g_{-1, j}(\lambda, \partial)=(j+1) \lambda \text { for } 1 \leq j \in \mathbb{Z}, \\
& g_{j, i}(\lambda, \partial)=(i+j+2) \lambda+(j+1) \partial \text { for } 1 \leq i \in \mathbb{Z}, 1 \leq j \in \mathbb{Z} .
\end{aligned}
$$

Using Lemma 4.1 and the fact that $\Delta_{j}=j+2$ for $1 \leq j \in \mathbb{Z}$, we can immediately obtain that the above two formulae hold for all $i, j \in \mathbb{Z}_{\geq-1}$. Therefore, we have proved that $g_{j, i}(\lambda, \partial)=(i+j+2) \lambda+(j+1) \partial$ for all $i, j \in \mathbb{Z}_{\geq-1}$, which is equivalent to that $\mathcal{G} \cong B(2,0)$.

Case 3: $\Delta_{-1}=1, \Delta_{1}=3, \alpha_{1} \neq 0$ and $g_{1,-1}(\lambda, \partial)=-\frac{a_{-1,1}^{0}}{\alpha_{1}}\left(\alpha_{1}-\lambda-\partial\right)$, where $a_{-1,1}^{0} \in \mathbb{C}^{*}$.

In this case, by skew-symmetry, we get $g_{-1,1}(\lambda, \partial)=a_{-1,1}^{0}\left(1+\frac{1}{\alpha_{1}} \lambda\right)$ and $g_{1,0}(\lambda, \partial)=$ $-\alpha_{1}+3 \lambda+2 \partial$. Then (5.2) leads to

$$
g_{-1,2}(\lambda, \partial) g_{1,1}(\mu, \lambda+\partial)=3 a_{-1,1}^{0}(\lambda+2 \mu+\partial)\left(1+\frac{1}{\alpha_{1}} \lambda\right) .
$$

Setting $\lambda=0, \partial=0$ and $\lambda=\partial=0$ in the above formula respectively, noting that $g_{-1,2}(\lambda, \partial)$ and $g_{1,1}(\lambda, \partial)$ are polynomials of $\lambda$ and $\partial$, we obtain

$$
\begin{aligned}
& g_{-1,2}(\lambda, \partial)=a_{-1,2}^{0}\left(1+\frac{1}{\alpha_{1}} \lambda\right), \\
& g_{1,1}(\lambda, \partial)=\frac{3 a_{-1,1}^{0}}{a_{-1,2}^{0}}(2 \lambda+\partial),
\end{aligned}
$$

where $a_{-1,2}^{0} \in \mathbb{C}^{*}$. Taking $j=2, \Delta_{-1}=1$ and $\Delta_{1}=3$ in $(5.1)$, noting that $a_{-1,2}^{0} \neq 0$, we get

$$
\Delta_{2}=4
$$

By (4.10), (5.1) and (5.15)-(5.17), we can inductively deduce

$$
\begin{aligned}
& \Delta_{j}=j+2, \\
& g_{-1, j}(\lambda, \partial)=a_{-1, j}^{0}\left(1+\frac{1}{\alpha_{1}} \lambda\right), \\
& g_{1, j}(\lambda, \partial)=\frac{a_{-1,1}^{0}}{2 a_{-1, j+1}^{0}}(j+2)\left((j-1) \alpha_{1}+(j+3) \lambda+2 \partial\right),
\end{aligned}
$$

where $1 \leq j \in \mathbb{Z}$ and $a_{-1, j}^{0} \in \mathbb{C}^{*}$. Similar to Case 1, from (5.10) and the above three 
formulae, it inductively follows that

$$
\begin{aligned}
g_{j, i}(\lambda, \partial)= & \frac{a_{-1,1}^{0} a_{-1,2}^{0} \cdots a_{-1, i}^{0}}{a_{-1, j+1}^{0} a_{-1, j+2}^{0} \cdots a_{-1, j+i}^{0}} \times \frac{(j+2)(j+3) \cdots(j+i+1)}{(i+1) !} \\
& \times\left((i-j) \alpha_{1}+(j+i+2) \lambda+(j+1) \partial\right)
\end{aligned}
$$

for $1 \leq i \in \mathbb{Z}$ and $1 \leq j \in \mathbb{Z}$. Since in this case $\alpha_{1} \neq 0$, by replacing $\mathfrak{g}_{j}$ by $\mathfrak{g}_{j}^{\prime}=$ $\frac{(j+1) ! \alpha_{1}^{j}}{a_{-1,1}^{0} a_{-1,2}^{0} \cdots a_{-1, j}^{0}} \mathfrak{g}_{j}$ for $1 \leq j \in \mathbb{Z}$, we obtain that $g_{-1, j}(\lambda, \partial)$ and $g_{j, i}(\lambda, \partial)$ have the following forms,

$$
\begin{aligned}
& g_{-1, j}(\lambda, \partial)=(j+1)\left(\alpha_{1}+\lambda\right) \text { for } 1 \leq j \in \mathbb{Z}, \\
& g_{j, i}(\lambda, \partial)=(i-j) \alpha_{1}+(i+j+2) \lambda+(j+1) \partial \text { for } 1 \leq i \in \mathbb{Z}, 1 \leq j \in \mathbb{Z} .
\end{aligned}
$$

By Lemma 4.1 and noting that $\Delta_{j}=j+2$ for $1 \leq j \in \mathbb{Z}$, we can immediately conclude that the above two formulae hold for all $i, j \in \mathbb{Z}_{\geq-1}$. Hence, we obtain that $g_{j, i}(\lambda, \partial)=$ $(i-j) \alpha_{1}+(i+j+2) \lambda+(j+1) \partial$ for all $i, j \in \mathbb{Z}_{\geq-1}$ and $\alpha_{1} \neq 0$. It follows that in this case, we have $\mathcal{G} \cong B(2, \alpha)$ for some $\alpha \in \mathbb{C}^{*}$.

Therefore, the above three cases together show that Theorem 1.3 (3) holds.

\section{References}

[1] C. Boyallian, V. Kac, J. Liberati, Finite growth representations of infinite Lie conformal algebras, J. Math. Phys. 44(2003), no. 2, 754-770.

[2] C. Boyallian, V. Kac, J. Liberati, On the classification of subalgebras of $\operatorname{Cend}_{N}$ and $g c_{N}$, J. Algebra 260(2003), 32-63.

[3] B. Bakalov, V. Kac, A. Voronov, Cohomology of conformal algebras, Comm. Math. Phys. 200 (1999), 561-598.

[4] A. Belavin, M. Polyakov, B. Zamolodchikov, Infinite conformal symmetry in two-dimensional quantum field theory, Nucl. Phys. B 241 (1984), 333-380.

[5] C. Boyallian, V. Kac, J. Liberati, A. Rudakov, Representations of simple finite Lie conformal superalgebras of type $W$ and S, J. Math. Phys. 47 (2006), no. 4, 043513.

[6] S. Cheng, V. Kac, Conformal modules, Asian J. Math. 1 (1997), no.1, 181-193. Erratum 2(1998), no. 1, 153-156.

[7] S. Cheng, V. Kac, M. Wakimoto, Extensions of conformal modules, Topological field theory, primitive forms and related topics, Proceedings of Taniguchi symposia, Progress in Math., Birkhauser. 
[8] A. D'Andrea, V. Kac, Structure theory of finite conformal algebras, Sel. Math. (N.S.) 4 (1998), no. 3, 377-418.

[9] A. De Sole, V. Kac, Lie conformal algebra cohomology and the variational complex, Comm. Math. Phys. 292 (2009), 667-719.

[10] D. Fattori, V. Kac, Classification of finite simple Lie conformal superalgebras, Special issue in celebration of Claudio Procesi's 60th birthday, J. Algebra 258 (2002), no. 1, 23-59.

[11] D. Fattori, V. Kac, A. Retakh, Structure theory of finite Lie conformal superalgebras, Lie theory and its applications in physics V, (2004), 27-63, World Sci. Publ., River Edge, NJ.

[12] M. Gao, Y. Xu, X. Yue, The Lie conformal algebra of a Block type Lie algebra, Algebra Colloquium, 22(3) (2015), 367-382.

[13] P. Kolesnikov, On finite representations of conformal algebras. J. Algebra, 331 (2011), 169193.

[14] P. Kolesnikov, The Ado theorem for finite Lie conformal algebras with Levi decomposition, J. Algebra Appl., 15(7) (2016), 1650130.

[15] V. Kac, Vertex algebras for beginners, University Lecture Series 10 (1998), American Mathematical Society.

[16] V. Kac, The idea of locality, in: H.-D. Doebner et al (eds), Physical applications and mathematical aspects of geometry, groups and algebras (1997), 16-32, World Sci., Singapore.

[17] V. Kac, Lie conformal algebras, Resenhas 6 (2004), 223-230.

[18] Y. Su, Low dimensional cohomology of general conformal algebras $g c_{N}$, J. Math. Phys. 45 (2004), 509-524.

[19] Y. Su, C. Xia, L. Yuan, Classification of finite irreducible conformal modules over a class of Lie conformal algebras of Block type, J. Algebra 499 (2018), 321-336.

[20] Y. Su, X. Yue, Filtered Lie conformal algebras whose associated graded algebras are isomorphic to that of general conformal algebra $g c_{1}$, J. Algebra 340 (2011), 182-198.

[21] H. Wu, Q. Chen, X. Yue, Loop Virasoro Lie conformal algebra, J. Math. Phys. (2014), 011706.

[22] E. Zelmanov, On the structure of conformal algebras, Combinatorial and computational algebra (Hong Kong, 1999), 139-153, Contemp. Math., 264, Amer. Math. Soc., Providence, RI, 2000. 